\title{
Efecto de Pyraclostrobin + Epoxiconazole en la producción de fresa (Fragaria sp.)
}

\section{Effect of applying Pyraclostrobin + Epoxiconazole in the strawberry production (Fragaria sp.)}

Marco Alexander Luis-Ayala', Pedro José Almanza-Merchan², Pablo Antonio Serrano-Cely ${ }^{3}$

\section{RESUMEN}

Cundinamarca ha sido un gran productor de fresa(Fragaria sp),en comparación con los demás departamentos de Colombia; pero uno de sus mayores limitantes es la pérdida de fruta causada por el moho gris, enfermedad desarrollada por el agente Botritis cinerea; por esta razón, se aplicó Pyraclostrobin+Epoxiconazole ${ }^{\circledR}$ a los cultivos, en tres dosis, y se compararon con un testigo regional, Carbendazim ${ }^{\circledR}$, teniendo como variables de estudio: producción, calidad, grados Brix, $\mathrm{pH}$, acidez titulable y la incidencia de la enfermedad sobre el cultivo. Los resultados obtenidos y analizados por medio del software SAS y aplicando las pruebas de comparación de Tukey demostraron que la dosis de Pyraclostrobin + Epoxiconazole ${ }^{\circledR}$ de $80 \mathrm{cc} / 200 \mathrm{~L}$ presentó la mayor producción de fruta de segunda, tercera y total, y la menor cantidad de fruta dañada, junto con la dosis de

\begin{abstract}
Cundinamarca has been abig producer of strawberry (Fragaria sp.) in contrast to the other departments of Colombia. Yet one of its major constraints is the lost fruit caused by the gray mold disease, developed by the Botrytis cinerea agent. Therefore, it was applied Pyraclostrobin + Epoxiconazole ${ }^{\circledR}$, to the plantations, in three doses which were compared with a regional witness Carbendazim ${ }^{\circledR}$, with the study variables: production, quality, brix degrees, $\mathrm{pH}$, titratable acidity and the disease incidence on the crop. The results obtained and analyzed by SAS software and applying the Tukey comparison test, showed that the dose of pyraclostrobin + epoxiconazole with $80 \mathrm{cc} / 200 \mathrm{~L}$, had the highest fruit production of second, and third class, and as a whole. Aldo the lowest total amount of fruit damaged, along with the dose of $120 \mathrm{cc} / 200 \mathrm{~L}$. While the lower total
\end{abstract}

Profesional independiente. Correo elctrónico: aalexander531@gmail.com

Ph.D. Universidad Pedagógica y Tecnológica de Colombia (Tunja, Boyacá, Colombia). Correo electrónico: pedro. almanza@uptc.edu.co

3 M.Sc. Universidad Pedagógica y Tecnológica de Colombia (Tunja, Boyacá, Colombia). Correo electrónico: pablo. serrano@uptc.edu.co 
$120 \mathrm{cc} / 200 \mathrm{~L}$. La menor producción total y por calidad se obtuvo con la dosis de Carbendazim ${ }^{\circledR}$ (250cc/200L). En cuanto a los grados Brix, no se encontraron diferencias significativas, pero sí un aumento durante las cuatro semanas de cosecha. Los valores más altos de $\mathrm{pH}$ y ATT se obtuvieron con las dosis de Pyraclostrobin + Epoxiconazole ${ }^{\circledR}$, y los menores valores, con las aplicaciones de Carbendazim $^{\circledR}(250 \mathrm{cc} / 200 \mathrm{~L})$.Se recomienda hacer aplicaciones de estos ingredientes en el manejo de la enfermedad moho gris.

Palabras clave: Brix, Fresa, Fungicidas, Moho gris. production and quality was obtained with the dose of carbendazim (250cc/200L).In terms of brix degrees, no significant differences were found, but an increase over the four crop weeks. The $\mathrm{pH}$ and ATT highest values, were obtained with doses of pyraclostrobin + epoxiconazole ${ }^{\circledR}$ and the lowest values with applications of carbendazim (250 $\mathrm{cc} / 200 \mathrm{~L})$. It is recommended these ingredients applications in the management of gray mold disease (Botrytis cinerea).

Key Words: Brix, Strawberry, Fungicides, Gray mold. 


\section{INTRODUCCIÓN}

Colombia, por sus condiciones climáticas y edáficas, reúne las características para producir gran diversidad de cultivos, de los cuales se destacan los frutales y las hortalizas; en el año 2011, el país contaba con un área cosechada en fresa de 1.133,92 ha, con una producción de $40.000 \mathrm{t}$ y un rendimiento de 40 tha $^{-1}(1)$.

Cundinamarca ha sido conocido como gran productor de fresa, en contraste con los demás departamentos de Colombia; cuenta con un área cosechada de 700ha, con una producción de $30.000 \mathrm{t}$, y un rendimiento de 48 tha $^{-1}$. Según el porcentaje de participación por departamentos, Cundinamarca alcanzó en el 2011 un nivel de participación de 63,4\%, seguido de Norte de Santander (7\%), Cauca (3.6\%) y Boyacá (1.6\%) (1). En cuanto a los municipios productores de fresa en Cundinamarca, Chocontá participo con el $4.5 \%$ de la producción departamental, contando con un área sembrada en fresa de 81,80 ha, con un área cosechada de 56,80ha y un rendimiento de 50 tha1, para una producción de 2.840 t (1).

De esta forma, el municipio de Chocontá participa con el $11.86 \%$ de la producción de fresa del departamento de Cundinamarca para ese año; esto significa que este cultivo es de mucha importancia en el municipio y que, en consecuencia, se debe brindar al agricultor herramientas que le permitan aumentar la producción y la calidad de la fresa, así como establecer un manejo adecuando sobre la enfermedad moho gris (Botritis cinerea) (2, 3, 4). Las estrobirulinas, existentes en el mercado son derivadas sintéticas de productos naturales que actúan a través de inhibición de la respiración mitocondrial del hongo. Además del efecto fungicida, las estrobirulinas han promovido alteraciones fisiológicas en varios cultivos, que influencia positivamente en el rendimiento de granos y frutos $(5,6,7)$.

La Pyraclostrobina es una de las estrobirulinas más investigadas en el Brasil, en cuanto a su acción fungitóxica y de los efectos fisiológicos en el cultivo hospedero. Las primeras constataciones sobre el efecto fisiológico de esa estrobirulina fueron obtenidas en Alemania (8), en experimentos en los cultivos de trigo y cebada; se verificó que promovía alteraciones en el metabolismo y crecimiento de las plantas -efectos adicionales a la fisiología del cereal- y que aumentaba significativamente el rendimiento; además de su efecto fungicida, presenta efectos fisiológicos marcados en varios cultivos, promoviendo y aumentando la actividad de la enzima nitrato reductasa, disminución de la respiración foliar, disminución de la producción de etileno, aumento de la síntesis de hormonas promotores del desenvolvimiento y aumento de la actividad de las enzimas peroxidasa y superóxidodismutasa $(9,10,11)$. Con la investigación se buscó establecer el efecto del pyraclostrobin sobre la producción y calidad de la fresa (Fragaria sp), en el municipio de Chocontá (Cundinamarca).

\section{MATERIALES Y MÉTODOS}

La investigación se llevó a cabo en la vereda Tejar del municipio de Chocontá, a una altura de 2655 msnm, con una temperatura promedio de $13^{\circ} \mathrm{C}$ y precipitación de $2.360 \mathrm{~mm} / \mathrm{año}$. El cultivo de fresa (Fragaria sp.) pertenece a la variedad monterrey, con un total de 150.000 plantas en un área de $10.000 \mathrm{~m}^{2}$. Para la investigación se tomaron 1560 plantas de un año de edad, las cuales se encontraban distribuidas en 12 surcos; se les realizó una poda, con el objetivo de permitir un rebrote y garantizar que todas se encontraran en el mismo estado fisiológico.

Se realizó un diseño en BCA, con 3 bloques, compuestos por 4 caballones cada uno, donde se ubicaron los cuatro tratamientos al azar; cada caballón contaba con 130 plantas. Para la distribución de los bloques se tuvo en cuenta tanto la pendiente y el suministro de fertirriego. Se realizaron aplicaciones de productos que tienen Pyraclostrobin como uno de sus ingredientes, ya que se quiere determinar el efecto propio de esta estrobirulina, y no de la rotación del producto comercial a base o en combinación de otro ingrediente; en este sentido, se realizó la respectiva comparación entre las diferentes dosis (Tabla I). 
Tabla I. Distribución de los tratamientos

\begin{tabular}{|c|c|c|c|}
\hline Tratamiento & Moléculas & Dosis & Dosis/tto. \\
\hline T1 & Piraclostrobin + Epoxiconazol & $80 \mathrm{cc} / 200 \mathrm{~L}$ & $6 \mathrm{cc} / 15 \mathrm{~L}$ \\
\hline T2 & Piraclostrobin + Epoxiconazol & $100 \mathrm{cc} / 200 \mathrm{~L}$ & $7,5 \mathrm{cc} / 15 \mathrm{~L}$ \\
\hline T3 & Piraclostrobin + Epoxiconazol & $120 \mathrm{cc} / 200 \mathrm{Lt}$ & $9 \mathrm{cc} / 15 \mathrm{~L}$ \\
\hline T4 & Carbendazim & $250 \mathrm{cc} / \mathrm{L}$ & $18,75 \mathrm{cc} / 15 \mathrm{~L}$ \\
\hline
\end{tabular}

La aplicación de Carbendazim se tomó como un testigo, ya que es el producto que se usa habitualmente en las rotaciones para el control de la pudrición gris (Botrytis cinerea); se realizó 1 aplicación semanalmente, una después de cada cosecha, para un total de 4 cosechas y aplicaciones. Los datos fueron registrados en un formato previamente elaborado.

La calibración de la bomba de espalda, con capacidad de 20L, se realizó llevándola a su volumen máximo con agua, luego se asperjó una de las hileras de fresa (Fragaria sp.), tomando el volumen inicial y final se extrapolaron las dosis del producto comercial al volumen de mezcla necesario para realizar la aplicación de los productos $\quad$ (Pyraclostrobin + Epoxiconazole ${ }^{\circledR}$ ) y Carbendazim $^{\circledR}$, necesitando un volumen de mezcla de $15 \mathrm{~L}$ por tratamiento.

\section{Determinación de las variables de estudio}

- Peso fresco. La determinación de esta variable se refiere al peso que se tomó en campo los días de cosecha, es decir, qué cantidad de fruta por tratamiento; el valor se estableció mediante peso directo en una balanza electrónica marca ADAM $^{\circledR}$ PGW2502e con capacidad máxima de 2500g $\mathrm{d}=0.01 \mathrm{~g}$.

- Calidad. Se evaluó de acuerdo con la cantidad de fruta y según sus características físicas; esta clasificación se realizó durante la cosecha, teniendo como referente lo citado por Angulo (2009) y con base en la norma técnica colombiana (10).

-Incidenciadelaenfermedad.Comolosingredientes aplicados, $\quad$ Pyraclostrobin + Epoxiconzole ${ }^{\circledR} \quad$ y Carbendazim $^{\circledR}$, son usados para el control de hongos fitopatógenos, en este caso se tomaron datos de la incidencia de Botrytis cinerea sobre el cultivo, pesando la cantidad de fresa afectada, así como la presencia en las plantas por tratamiento y dividiéndolo en el número total de plantas.

Variables fisicoquímicas. Se midieron cada una de las variables el mismo día de la cosecha, ya que se podían presentar cambios en algunas de ellas pasado el tiempo de cosecha; la determinación de la variables de laboratorio se determinaron con frutos con una maduración del $75 \%$, ya que es el punto óptimo de cosecha establecido por el productor, para que los frutos no sufran problemas en el transporte. Los frutos, una vez cosechados, se Ilevaron al laboratorio de fisiología vegetal de la Uptc, en donde se realizaron las pruebas fisicoquímicas.

- Grados Brix. Esta variable se determinó por medio de un refractómetro digital marca Hanna, de rango 0 a $85 \%$, con precisión $0,1{ }^{\circ}$ Brix; después de macerar una muestra representativa de fresas de cada tratamiento y dependiendo de la calidad $(1,2)$, se tomó una gota de jugo de los frutos; las fresas se tomaron con el mismo estado de madures 4.

- $\mathbf{p H}$. Se determinó por medio de un pH-metro digital, Metrohm. Esta medición se realizó después de cada cosecha, junto con los grados Brix, aprovechando el jugo producto de la maceración de las fresas.

- Acidez titulable. Se determinó pesando $5 g$ de jugo de fresa en una balanza digital ADAM $^{\circledR}$ PGW2502e con capacidad máxima de 2500g $d=0.01 \mathrm{~g}$. Luego, se agregaron 3 gotas del indicador fenoftaleina y se procedió a la titulación con $\mathrm{NaOH} 1 \mathrm{~N}$ por medio de una bureta digital Titrette $^{\circledR}$. Luego de esto se procedió al cálculo 
por medio de la fórmula sugerida por la Norma Técnica Colombiana 4103 (10), realizada para fresa Chandler, la cual dice que ATT $=$ (Volumen $\mathrm{NaOH} \times \mathrm{N}$ de $\mathrm{NaOH} \times \mathrm{O} .064 \mathrm{~g} / \mathrm{meq}) / 5 \mathrm{ml}$.

Análisis estadístico. Se realizó un análisis estadístico de forma cuantitativa, determinando las diferencias existentes entre cada uno de los tratamientos (T1, T2, T3 y T4) con respecto a cada variable (Brix, pH, acidez titulable, producción, calidad e incidencia de moho gris), durante las 4 semanas de cosecha; los datos tomados tanto en campo como en laboratorio fueron analizados estadísticamente por medio de software SAS, y también se llevó a cabo la prueba de comparación múltiple de Tukey (5\%) para determinar la diferencia de los tratamientos frente a cada una de las variables.

\section{RESULTADOS Y DISCUSIÓN}

\section{Comportamiento de la producción}

- Producción total. Se presentaron diferencias significativas en la producción total de las calidades segunda, tercera, dañada y total; en la calidad primera no se observaron diferencias estadísticas. La aplicación de Pyraclostrobin + Epoxiconazole ${ }^{\circledR}$ (80cc/200L) se caracterizó por generar la mayor producción total de las calidades segunda, tercera y total, y la dosis que presentó menor cantidad de fruta dañada fue Pyraclostrobin + Epoxiconazole 120cc/200L. De forma contraria, la aplicación de Carbendazim ${ }^{\circledR}$ fue responsable de la menor producción de frutos de fresa de las calidades segunda, tercera y total, y la mayor cantidad de frutos dañados (Figura 1).

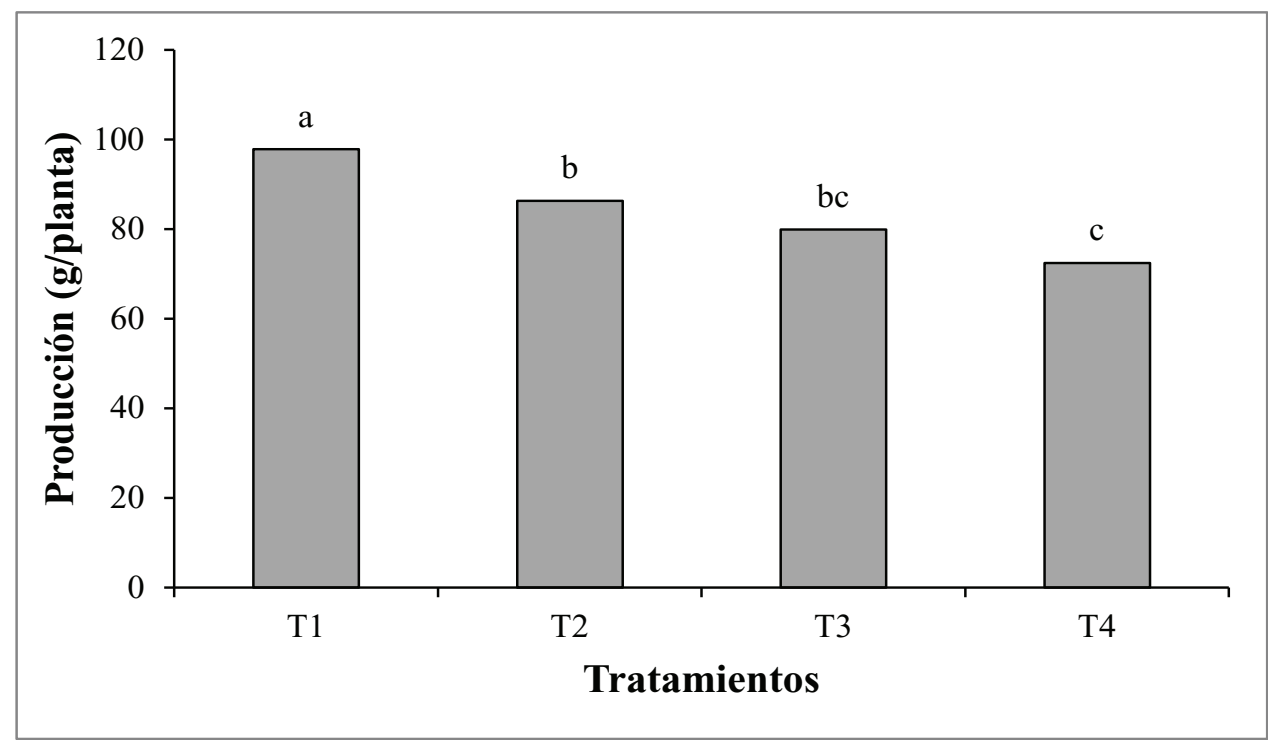

Figura 1. Efecto de la aplicación de Pyraclostrobin + Epoxiconazol ${ }^{\circledR}$ en la producción total de frutos de fresa (Fragaria sp.) en el municipio de Chocontá. Letras diferentes en cada punto de muestreo presentan diferencias significativas $(p<0,05)$.

La menor producción total de fruta dañada se obtuvo con los tratamientos T1 y T3; la mayor producción total de fresa, durante las cuatro semanas de cosecha, se obtuvo con $\mathrm{T} 1$, correspondiente a la dosis más baja de Pyraclostrobin + Epoxiconazol ${ }^{\circledR}$ (80cc/200L), es decir, que el aumento en la producción no está relacionado del todo con el control de la enfermedad, ya que con T2 se obtuvo una producción total por debajo de T1, pero por mayor a la de T3, lo que coincide con varios investigadores que reportan que el Pyraclostrobin tiene efectos bioestimulantes en el metabolismo vegetal $(11,12)$.

Los datos también coinciden con lo reportado en otros cultivos, como avena, cebada, fríjol, maíz, soya, trigo y frutales $(13,14,15)$, en cuyo rendimiento se ha visto el efecto del Pyraclostrobin, asociado a la activación de la enzima NADHnitrato reductasa, la cual se relaciona con el 
aumento de la eficiencia en la asimilación de nitrógeno y de $\mathrm{CO}_{2}$, así como con el incremento en la tasa fotosintética y con la reducción de la tasa respiratoria (9).

En cuanto a la producción total en términos de rendimiento, los datos anteriores concuerdan con lo citado por Fischer (13), quien reporta que el periodo vegetativo de la fresa en el país es de 18 a 24 meses, durante los cuales cada planta produce entre 1000 y 2000g, lo cual depende de la variedad utilizada y de un correcto manejo agronómico.
En este sentido, la producción total durante las cuatro semanas de cosecha fue, en promedio, de 85g/planta, y llevando esta producción a un año tendríamos 1020g/planta.

\section{Calidad interna de los frutos}

- Sólidos Solubles Totales (SST). Se observó un leve aumento de los SST en función del tiempo de cosecha, pero no se presentaron diferencias estadísticas; los valores oscilaron entre 5,92 y $6,23^{\circ} \mathrm{Bx}$ (Figura 2).

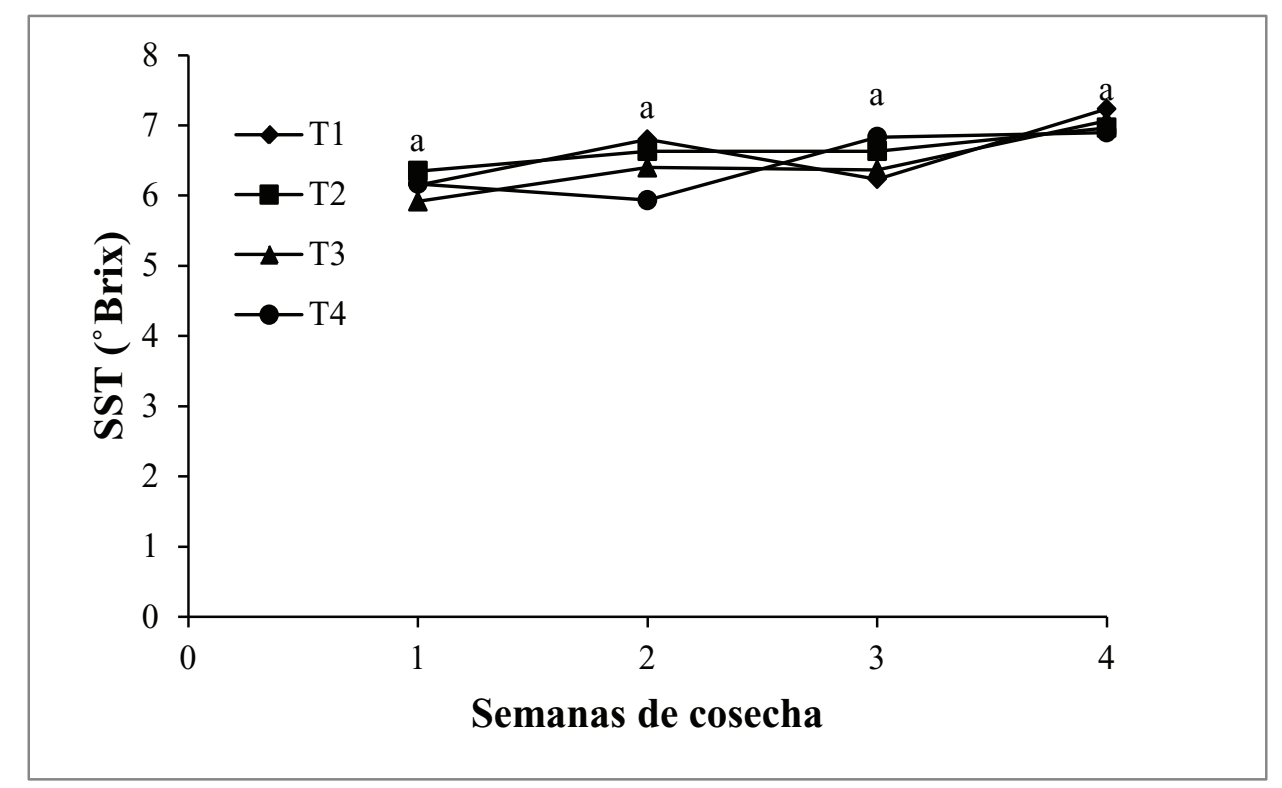

Figura 2.Sólidos Solubles Totales (BRIX) en el municipio de Chocontá

Letras diferentes en cada punto de muestreo presentan diferencias significativas $(p<0,05)$.

Los resultados anteriores difieren un poco con lo reportado por Angulo (9), ya que si se presenta un aumento en la actividad de la NR, el proceso de asimilación de $\mathrm{N}$, en forma $\mathrm{NO}_{3}^{-}$, y su paso a $\mathrm{NO}_{2}$, como resultado de la intervención de $\mathrm{NR}$, y posteriormente de la NIR, para ser trasformado en $\mathrm{NH}_{4}{ }^{+}$, el exceso de $\mathrm{N}$ se va a manifestar en aumento de los grados Brix de los frutos de fresa; sin embargo, todo esto estaría sujeto al manejo de la fertilización y a la disponibilidad de nutrientes, ya que como el manejo de la fertilización en el cultivo se hace por medio del fertirriego, las sales tienden a acumularse, creando problemas de $\mathrm{CIC}$ del suelo.
Por otra parte, Barahona y Sancho (16) aseguran que el contenido nutritivo y los grados Brix del fruto varían según la variedad, la fertilidad de los suelos y las condiciones climáticas, principalmente. En este sentido, los datos anteriores concuerdan con lo reportado por Torres (17) quien tras aplicar tres dosis de Pyraclostrobin aun cultivo de naranja no observó efecto sobre la producción, pues los grados Brix no aumentaron con las aplicaciones de Pyraclostrobin con respecto al testigo; sin embargo, difieren de los reportado por Rivas (15), quien al hacer tres aplicaciones de Pyraclostrobin+Boscalid ${ }^{\circledR}$, en dosis de $800 \mathrm{~g} /$ ha, en un cultivo de melón cantaloupe, variedad 
Caribbean Gold, obtuvo un aumento de los grados Brix con respecto al testigo. Datos similares reportó Solís (18), quien al realizar aplicaciones de Pyraclostrobin + Boscalid $^{\circledR}$ para determinar el efecto sobre los grados Brix y la vida de anaquel en el cultivo de zanahoria (Daucus carota), obtuvo como resultado un aumento en los grados Brix, 7.5 y 7 , con respecto al testigo.

Por su parte, Pascoe (19) reporta que el parámetro de grados Brix, durante el desarrollo de un ensayo donde se realizó la aplicación de Pyraclostrobin + Boscalid $^{\circledR}$ como promotor de vida de anaquel en zarzamora de exportación, no aportó mucha información debido a que es un valor muy ligado a la nutrición; se puede tener fruta de buena calidad, pero muy ácida. Lo anterior concuerda con los resultados obtenidos a los largo del presente estudio.

Otros resultados, obtenidos por Acevedo (20), al realizar la evaluación de Pyraclostrobin +
Epoxiconazole $^{\circledR}$ en la producción y calidad del espárrago UC 157 F1, en la localidad de Ica (Perú), demuestra un aumento de los grados Brix al hacer dos aplicaciones en la dosis $0.75 \mathrm{~L} / \mathrm{ha}$.

- pH. El pH tuvo el mayor valor en la semana 3 , y solo se presentaron diferencias estadísticas en la semana 2, en este punto; los frutos de fresa con menor $\mathrm{pH}$ fueron los tratados con Piraclostrobin + Epoxiconazol ${ }^{\circledR} \quad(120 \mathrm{cc} / 200 \mathrm{Lt})$, pero con Carbendazim $^{\circledR} \quad(250 \mathrm{cc} / 200 \mathrm{~L})$ se obtuvieron los frutos con mayor pH (Figura 3).

Según Nanos y Kader (21), citados por Balaguera (22), en frutos, más de $90 \%$ del volumen celular lo ocupa la vacuola, que, generalmente, es muy ácida, con $\mathrm{pH}$ inferior a 5, situación que coincide para los frutos de fresa; sin embargo, como se observa en la Figura 3, los T1, T2, y T3, correspondientes a las dosis de Pyraclostrobin + Epoxiconazole ${ }^{\circledR}$, presentan los valores más bajos con relación al testigo.

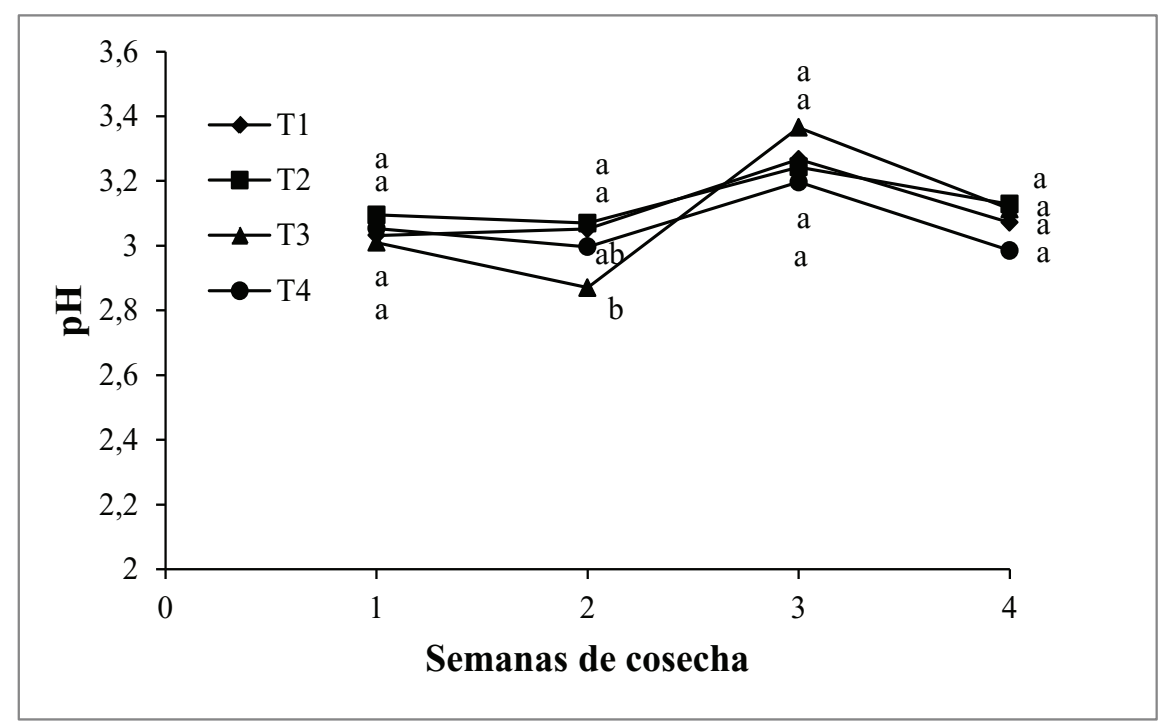

Figura3. Efecto de la aplicación de Pyraclostrobin + Epoxiconazo ${ }^{\circledR}$ en el pH de frutos de fresa (Fragaria sp.) en el municipio de Chocontá. Letras diferentes en cada punto de muestreo presentan diferencias significativas $(p<0,05)$.

De acuerdo con lo anterior, el bajo $\mathrm{pH}$ puede estar asociado con dos aspectos: 1) altos contenidos de ácidos almacenados por la vacuola y 2) el crecimiento de las células, que necesitan bajos niveles de $\mathrm{pH}$ (18). En relación con lo anterior y según lo citado en otras investigaciones $(19,20,21$,
22, 23, 25), dentro de los efectos bioestimulantes del Pyraclostrobin se destaca el aumento en la biomasa; en este sentido, dicho efecto podría disminuir el $\mathrm{pH}$, teniendo así resultados por debajo del testigo. 
Por otra parte, la poca diferencia significativa durante las cuatro semanas de cosecha puede estar relacionada con la presencia de un sistema de autorregulación del $\mathrm{pH}$, que podría ser el resultado de un efecto amortiguador, o buffering, del ácido cítrico, como ya ha sido reportado por Dunne (24); sin embargo, esto no concuerda con lo reportado por Menéndez et al. (26), citado por Balaguera (22); este ácido alifático tiende a convertirse en la sal correspondiente, dando como resultado el efecto amortiguador de la solución en la región de sus valores de $\mathrm{pKa}$, manteniendo constante el valor del $\mathrm{pH}$, pero disminuyendo la acidez, con relación a la disminución de la ATT, ya que en el presente estudio la ATT aumentó de forma leve, lo que permite intuir que este leve aumento en la acidez total titulable pudo deberse a un aumento en la concentración de ácido cítrico, el cual hizo que el $\mathrm{pH}$ se mantuviera casi constante.

Por otra parte, según Rodríguez et al. (27), citado por Balaguera (22), el valor del $\mathrm{pH}$ es importante cuando el fruto va a ser industrializado, porque está relacionado con los costos de transformación del producto. En guayaba se ha encontrado que frutos con un nivel de $\mathrm{pH}$ de alrededor de 3,4, y menores valores de acidez, implican mayor economía en el proceso, porque hay que adicionar menos ácidos para la corrección del pH (25); este autor también mencionan que a mayor acidez existe mayor posibilidad de industrialización de los frutos en forma de dulces. Consecuentemente, Andrade et al. (28) afirman que para la elaboración de jugos, un alto nivel de acidez provoca una elevada dilución del producto y, por consiguiente, un aumento en el rendimiento final.

- Acidez Total Titulable (ATT). La ATT incrementó de forma leve durante las semanas de cosecha para la mayoría de los tratamientos (Figura 4), con excepción de los frutos tratados con Carbendazim $^{\circledR} \quad(250 \mathrm{cc} / 200 \mathrm{~L})$,los cuales presentaron una disminución en la semana 4. Se presentaron diferencias estadísticas en las semanas 3 y 4 ; en esta última, el menor valor de ATT se obtuvo en los frutos con Carbendazim ${ }^{\circledR}$, y el mayor valor se logró con la aplicación de Piraclostrobin + Epoxiconazol ${ }^{\circledR}(80 \mathrm{cc} / 200 \mathrm{Lt})$.

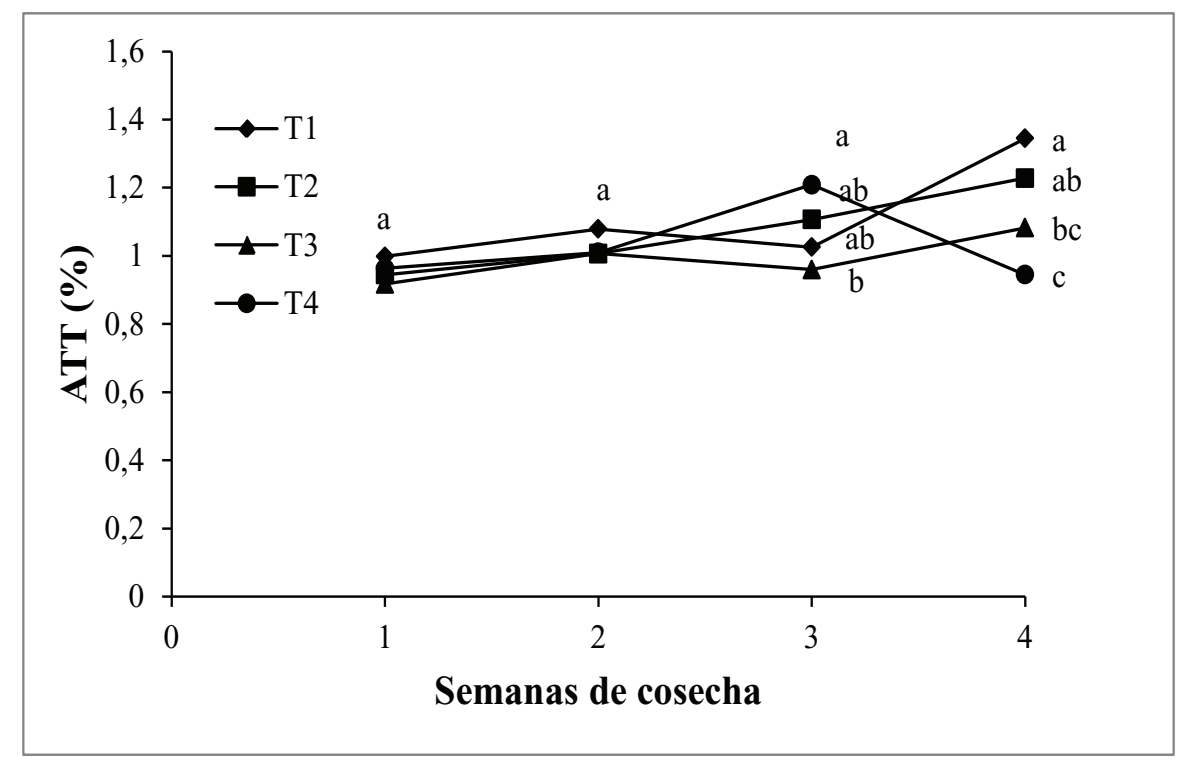

Figura 4. Efecto de la aplicación de Pyraclostrobin + Epoxiconazol ${ }^{\circledR}$ en la Acidez Total Titulable de frutos de fresa (Fragaria sp.) en el municipio de Chocontá. Letras diferentes en cada punto de muestreo presentan diferencias significativas $(p<0,05)$. 
Los análisis químicos de $\mathrm{pH}$, acidez total titulable y solidos solubles totales son comúnmente realizados en la mayoría de frutos, pues están directamente relacionados con el sabor presentado (Silva et al) (29); las concentraciones presentadas pueden influenciar la aceptación del fruto por el consumidor. Chitarra y Chitarra (30) mencionan que con la maduración, los frutos pierden rápidamente la acidez, pero en algunos casos hay un pequeño aumento; esto puede ser usado, en conjunción con la dulzura, como punto de referencia de la madurez.

Por otra parte, en feijoa se estableció un aumento del contenido de ácidos orgánicos, similar a lo reportado en guayaba, en la que la acidez titulable aumentó progresivamente hasta la cosecha (26). Lo anterior concuerda con los datos obtenidos en esta investigación, ya que los frutos de fresa cosechados durante las cuatro semanas fueron tomados en un estado 4, del $75 \%$ de maduración, es decir, que los frutos podrían aumentar de forma leve la ATT, según la cantidad de ácido cítrico que presenten.

Incidencia de moho gris (Botrytis cinérea) .Como se observa en la Figura5, durante las semanas 2 y 3 se presenta un aumento en el porcentaje de incidencia de la enfermedad moho gris, cuyo agente causal es $B$. cinerea; durante estas dos semanas, después de unos días de verano se presentan algunas precipitaciones, con lo cual se crea un medioambiente adecuado para la presencia de dicha enfermedad; sin embargo, el mejor control sobre la enfermedad en las semanas 2 y 3 se obtuvo con el tratamiento T2, es decir, la dosis de $80 \mathrm{cc} / 200 \mathrm{~L}$, seguido, en su orden, por los tratamientos T3, T1 y T4. T3, siendo la dosis más alta de Pyraclostrobin + Epoxiconazole ${ }^{\circledR}$, no demuestra tener el mayor control sobre la enfermedad, y el testigo regional T4 $\left(\right.$ Carbendazim $\left.{ }^{\circledR}\right)$ tiene un control sobre la enfermedad, pero parece estandarizarse entre las semanas 4 y 5 , al igual que el tratamiento T2, que para la semana 5 es superado por T3.

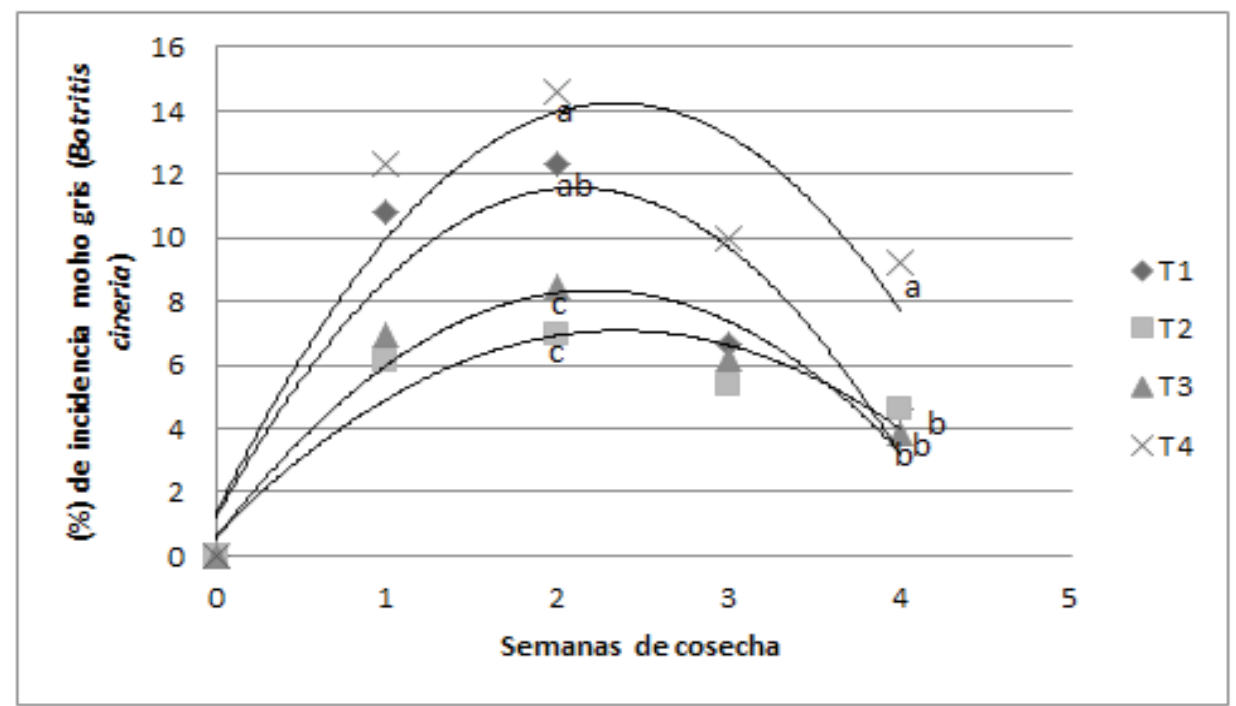

Figura 5. Efecto de la aplicación de Pyraclostrobin + Epoxiconazol ${ }^{\circledR}$ en el control de Moho Gris (Botritis cinerea) en los frutos de fresa (Fragaria sp.) en el municipio de Chocontá. Letras diferentes en cada punto de muestreo presentan diferencias significativas $(p<0,05)$.

Con relación a los datos anteriores, el control del moho gris (B. cinerea)bajo las tres dosis de Pyraclostrobin + Epoxiconazo ${ }^{\circledR}$, con respecto al testigo, se debe a que el Pyraclostrobin hace parte del grupo de los inhibidores de la quinona oxidasa (Qol), cuya toxicidad proviene de la inhibición de la cadena respiratoria (27); en el complejo III, citocromo b, ubicado dentro del complejo citocromo bc1 (18), impide la cadena bioquímica de trasferencia de electrones (25), inhibe la producción de ATP y genera estrés oxidativo por escape de electrones de la cadena respiratoria 
mitocondrial; de esta manera impide el desarrollo del patógeno (19).La influencia transitoria en las mitocondrias de las plantas no resulta necesariamente en fitotoxicidad, debido a que la toxicidad a nivel de organismo está determinada por la importancia de la respiración mitocondrial para el suministro de energía (23).

\section{CONCLUSIONES}

Se demuestra que la dosis de Pyraclostrobin + Epoxiconazole ${ }^{\circledR}$ de $80 \mathrm{cc} / 200 \mathrm{~L}$ presenta la mayor producción de fruta de segunda, tercera y total, y la menor cantidad de fruta dañada, junto con la dosis de 120cc/200L; la menor producción total y por calidad se obtuvo con la dosis de Carbendazim ${ }^{\circledR}(250 \mathrm{cc} / 200 \mathrm{~L})$. En cuanto a los grados Brix, no se encontraron diferencias significativas, pero sí un aumento durante las cuatro semanas. Los valores más altos de $\mathrm{pH}$ y ATT se obtuvieron con las dosis de Pyraclostrobin+Epoxiconazole ${ }^{\circledR}$, y los menores valores, con las aplicaciones de Carbendazim ${ }^{\circledR}$ (250 cc/200L).

\section{REFERENCIAS}

(1) Agronet. Anuario Estadístico de Frutas y Hortalizas 2007-2011 y sus calendarios de siembras y cosechas. Resultados evaluaciones agropecuarias municipales. Ministerio de Agricultura y Desarrollo rural. Colombia. 2011.

(2) Köehle, H., Gold R.E, Ammermann E, Sauter H, Roehl F. Biochem.Soc.Trans 1994; 22:65-73.

(3) Dourado D, Oliveira R, Begliomini E, RodriguesM. F500 em soja e milho. Atualidades Agrícolas BASF S.A 2005; 12-16.

(4) Arjona $\mathrm{H}$. Efecto bioestimulante Del pyraclostrobin.Arquivos do Instituto Biológico 2005; 72 (2): 234-41.

(5) Töfoli J.G, Domingues R.J, Garcia Júnior O. Controle da requeima do tomateiro com fungicidas e seus reflexos na produção. Arquivos do Instituto Biológico 2003; 70(4):473-82.

(6) Habermeyer J, Gerhard M, Zinkernagel V. The impact of strobilurins on the plant physiology of wheat. In: 7th International Congress of Plant Pathology, Glasgow, Scotland. Br.Soc. PlantPathol.Abstr 1998;5: 6-7.
(7) Vilariño M, Miralles D. Incrementos en la duración del área foliar en soja con la aplicación de Pyraclostrobin más Epoxiconazole en Ilenado de granos. Impacto sobre el rendimiento y sus componentes. 2005.

(8) Loayza F, Castro H. Evaluación de la aplicación de pyraclostrobin + epoxiconazole en la producción de maíz amarillo duro (dekalb 7088), bajo condiciones de la costa central de Perú. 2010.

(9) Angulo R. Fresa Fragaria ananassa. Produmedios. 2006.

(10) NORMA TÉCNICA COLOMBIANA, NTC, 4103. Segunda Actualización. Frutas y hortalizas frescas. 2011.

(11) Michael, G. The influence of strobilurine containing fungicides on physiological processes of yield formation in winter wheat varieties. Thesis, Berlin Technical University.2002.

(12) Fagan, E.A. cultura da soja: modelo de crescimento e aplicação da estrobilurina Piraclostrobina. Tesis de doctorado: Escola Superior de Agricultura Luiz de Queiroz. Piracicaba, Brasil. 2007.

(13) Fischer, G. Manual para el cultivo de frutales en el trópico, Produmedios, Bogotá.2012.

(14) Guzmán, H. Más es posible, hacemos rentable su negocio, BASF. Top Ciencia Revista 2011; 2: 1321.

(15) Rivas, J. Aplicaciones depyraclostrobin + Boscalid ${ }^{\circledR}$ evaluando el rendimiento y la calidad del cultivo de melón cantaloupe, variedad Caribbean Gold. 2011.

(16) Barahona, M.C. y Sancho, E. Manzana, melocotón, fresa y mora. Fruticultura especializada. Fruticultura II Universidad Estatal a distancia. San José, Costa Rica. 1998.

(17) Torres, B. Evaluación del efecto del Phyraclostrobin (Regnum ${ }^{\circledR}$ ) sobre la producción en el cultivo de naranja. 2011.

(18) Sólis, G. Efecto del AgCelence ${ }^{\circledR}$ de bellis ${ }^{\circledR} 38$ WG sobre los grados brix y vida de anaquel en el cultivo de zanahoria (Daucus carota). 2011.

(19) Pascoe L. Parámetros de grados brix durante el desarrollo de un ensayo en la aplicación de pyraclostrobin + Boscalid $^{\circledR}$ como promotor de vida de anaquel en zarzamora de exportación. 2009. 
(20) Acevedo O. Evaluación de pyraclostrobin + epoxiconazole en la producción y calidad del espárrago UC 157 F1 en la localidad de Ica, Perú. 2010.

(21) Nanos, G.D, Kader A.A. Low O2-induced changes in $\mathrm{pH}$ and energy charge in pear fruit tissue. Postharvest Biol. Technol 1993; 3: 285-291.

(22) Balaguera H. Estudio del crecimiento y desarrollo del fruto de champa (Campomanesia lineatifolia R\& P) y determinación del punto óptimo de cosecha. 2011.

(23) Salisbury F.B, Ross C.W. Fisiología de las plantas: desarrollo de las plantas y fisiología ambiental. Thompson Editores Spain, Paraninfo, Madrid. pp. 529-562. 2003.

(24) Dunne B. Strobilurin Use on Cereals. Crop Protection. 23:17-20. 2005.

(25)Venâncio W. Epoxiconazole: uma revição de uso, isolado ou em mistura, nas principais doencias da soja (Glicine max L.), feijão (Phaseolus vulgaris L.), amendoim (Arachis hypogaea L.), milho (Zea mays L.), trigo (Triticum aestivum L.), aveia (Avena spp.) e cevada (Hordeum vulgare L.). Universidade Estatal de Ponta Grossa. Ponta Grossa, Brasil. 2002.
(26)Menéndez O, Lozano S.E, Arenas M, Bermúdez K, Martínez A, Jiménez A. Cambios en la actividad de -amilasa, pectinmetilesterasa y poligalacturonasa durante la maduración del maracuyá amarillo (Passiflora edulis var. Flavicarpa degener). Interciencia 2006; 31(10): 728-733.

(27) Rodríguez M, Arjona H, Galvis J. Maduración del fruto de feijoa (Acca sellowiana Berg) en los clones 41 (Quimba) y 8-4 a temperatura ambiente en condiciones de Bogotá. Agronomía Colombiana 2006; 24(1):68-76.

(28)Andrade, J., Aragão, C. y Ferreira, S. Caraterización física y química de frutos de Araça-póra (Phidium acutangulum D.). Acta amazonica (Brasil) 23(2):213-217. 1993.

(29)Silva, E., Vilas Boas, E., Rodrigues, L. y Siqueira, H. Caracterización física, química y fisiológica de gariroba (Campomanesia pubecens) durante el desarrollo. Cienc. Tecnol. Aliment., Campinas 29(4):803-809. 2009.

(30)Chitarra M. y Chitarra, A. Pôs-colheita de frutos e hortaliças: fisiologia e manosciro. Lavras: ESALFAEPE, 2005. 